\title{
Selection of Cyanobacteria Grown In Wastewater Treatment Systems for Metabolites Production
}

\author{
Joan García \\ Universitat Politècnica de Catalunya-BarcelonaTech \\ Spain
}

Cyanobacteria (blue-green algae) are a photosynthetic procariotic group of microorganisms with a long adaptive and evolutionary diversification which has led to have a large and diverse array of metabolites with various bioactivities. In the last two decades the production of cyanobacteria has aroused special interest since they have been identified as one of the most promising group of organisms for the isolation of novel and biochemically active natural products. Unlike eukaryotic algae, cyanobacteria have the potential to assimilate and store compounds of interest such as glycogen and polyhydroxyalkanoates. Studies related to the production of cyanobacteria and their metabolites generally employ expensive pure or genetically modified cultures. An alternative approach for the production of cyanobacteria could be the use of wastewater-borne cyanobacteria cultures, using non-sterile waste streams as substrate. Wastewater treatment technologies are probably the most promising sustainable substitute to reduce additional production costs in cyanobacteria cultures. However, maintaining a dominant population of cyanobacteria in wastewater treatment systems is a challenging task. In this paper the factors determining the dominance of cyanobacteria are reviewed. Moreover, it is presented how to translate this knowledge on factors to cultures. Finally, strategies to obtain metabolites of interest are also reviewed. 